\title{
Positive and negative affect in cocaine use disorder treatment: Change across time and relevance to treatment outcome
}

\author{
Suzanne E. Decker, $\mathrm{PhD}^{1}$, Kristen P. Morie, $\mathrm{PhD}^{2}$, Bo Malin-Mayor, $\mathrm{BS}^{2}$, Charla Nich, MS $^{2}$, \\ and Kathleen M. Carroll, PhD $^{2}$ \\ ${ }^{1}$ New England Mental IIIness Research, Education, and Clinical Center and VA Connecticut \\ Health Care System, West Haven, Connecticut \\ ${ }^{2}$ Department of Psychiatry, Yale University School of Medicine, New Haven, Connecticut
}

\begin{abstract}
Background: Cognitive-behavior therapy (CBT) for substance use disorder is empirically supported, and may be associated with change in affect over time.
\end{abstract}

Objectives: Understanding the role of affect in cocaine use disorder could help to improve CBT outcomes.

\begin{abstract}
Methods: This secondary analysis included 140 adults with cocaine use disorder, 57.9\% female, drawn from two randomized controlled studies of web-based cognitive-behavior therapy who completed the Positive and Negative Affect Scale (PANAS) before and during treatment.
\end{abstract}

Results: In mixed-effects regression models, baseline negative affect (NA) scores were not
associated with self-reported cocaine use during treatment, but baseline PA scores were associated
with less frequent cocaine use $(\beta=-0.04, p=.02)$. During treatment, NA scores reduced over
time in CBT and treatment as usual $(\beta=-0.27, p<.01)$, although PA scores did not change.
Higher weekly NA scores were associated with weekly cocaine use $(\beta=0.02, \mathrm{SE}=0.01, t(746.15)$
$=2.37, p=.02)$, although weekly PANAS PA scores were not associated with weekly cocaine use.

Discussion and Conclusions: Results indicated that individuals with higher baseline PA were more likely to abstain from cocaine use during treatment, even when controlling for baseline cocaine use frequency. Although baseline NA was not associated with cocaine use, NA during treatment was associated with greater cocaine use.

Scientific Significance: PA at baseline and NA during treatment were associated with cocaine use. If findings are replicated, treatment developers may wish to include treatment interventions to boost early PA and reduce NA throughout treatment.

Cognitive-behavior therapy (CBT) is an empirically supported treatment for substance use disorder (SUD), including cocaine use disorder ${ }^{1}$. However, effect sizes for CBT for cocaine

Corresponding author: Suzanne E. Decker, PhD, 950 Campbell Avenue, 151D, West Haven, CT 06516, Tel: 203-932-5711 x7425, Fax: 203-937-3472, suzanne.decker@yale.edu.

Declaration of Interest: Dr. Carroll is a consultant to CBT4CBT LLC, which makes CBT4CBT available to qualified clinical providers and organizations on a commercial basis. Dr. Carroll works with Yale University to manage any potential conflicts of interest. The authors alone are responsible for the content and writing of this paper. Views expressed here are the authors' alone. 
use disorder remain modest (meta-analysis pooled effect size for cocaine, opioid, or stimulant use disorder $g=0.13,95 \%$ CI $[0.01,0.24])^{2}$. Understanding factors related to cocaine use during treatment may facilitate development of more effective treatments. One such potential factor is affect, which can be defined as two separate dimensions reflecting subjective distress and unpleasant engagement (negative affect; NA) and subjective pleasure, engagement, and energy (positive affect; PA) ${ }^{3}$. Within individuals with SUD, affect has been shown to vary when measured frequently (i.e., five times per day) ${ }^{4}$. However, high temporal stability in NA and PA ratings has indicated that even momentary affective states are, to some extent, indicative of a trait level of general affect ${ }^{3}$.

The literature suggests that both NA and PA may be implicated in SUD. High levels of NA and low levels of PA are thought to facilitate risky drug use ${ }^{5}$. NA may function as a relapse trigger, or individuals may use drugs to avoid the NA associated with withdrawal ${ }^{6}$. While this latter 'negative reinforcement theory' ${ }^{6}$ and the role of NA in addiction have been extensively studied, PA has been less frequently studied ${ }^{5}$. High PA may be a risk factor for the development of SUD, although low PA may also make an individual vulnerable to substance use ${ }^{5}$. Individuals may initially experience acutely increased PA immediately after drug use, but may become less sensitive to non-drug reinforcers that would otherwise elicit $\mathrm{PA}^{7}$. Across substances of abuse, low PA (anhedonia) has been reported during addiction ${ }^{5}$ and has also been described as a hallmark of withdrawal ${ }^{8}$. In cocaine use disorder, intoxication may include acutely elevated $\mathrm{PA}{ }^{9}$, while withdrawal may include dysphoria (elevated NA), possibly with anhedonia ${ }^{9}$. Anhedonia was also more prevalent among individuals with a remitted stimulant use disorder ${ }^{10}$. Given the complex relationships between NA and PA and substance use, further exploration of these relationships before and during SUD treatment is warranted and may lead to further avenues for improving existing SUD treatment.

Previous studies have used a variety of measures and timeframes for correlating affect and drug use. Young adults with cocaine dependence reported greater NA scores $(N=34, M=$ $21.38)$ on the PANAS ${ }^{3}$ compared with non-dependent cocaine users $(N=90, M=19.94)$ and with 'non-users' ( $N=883, M=18.29)$, but the groups did not differ on PA ${ }^{11}$. However, higher PA on the modified Differential Emotions Scale ${ }^{12}$ was indirectly associated with lower stimulant use among 88 treatment-seeking men ${ }^{13}$. Higher PA on the Ryman mood questionnaire ${ }^{14}$ was correlated with lower cocaine relapse likelihood in the next week in retrospective reports among 104 adults ${ }^{15}$. Within-person increases in past-hour boredom, anger, worry, discomfort, and sadness on a four-point Likert-type scale were reported prior to cocaine use in 112 treatment-seeking adults ${ }^{16}$.

Baseline predictors of SUD treatment outcome like drug-negative urine toxicology screens have been associated with end-of-treatment outcomes ${ }^{17}$. Consistent with prior study of affect in SUD ${ }^{13,15,16}$ and with theory ${ }^{5}$, baseline affect at the time of treatment-seeking may also be a potential treatment outcome predictor. Specifically, baseline NA may correlate with higher baseline cocaine use severity and future cocaine use. While less research exists to guide hypotheses about PA, available research suggests that baseline PA may negatively correlate with prior cocaine use severity and future cocaine use. 
An additional consideration is whether affect changes during SUD treatment. CBT for SUD helps clients identify situations or triggers, which could be external (e.g., people, places) or internal (e.g., changes in NA or PA) that could precede or follow cocaine craving or use ${ }^{18}$. CBT teaches clients to use drug refusal skills and to avoid decisions which could lead to substance use. Although clients exposed to CBT may be expected to experience reduced NA and increased PA as they learn to navigate situations that would otherwise lead to distress or drug use, few studies have examined affective changes during CBT. PANAS NA scores reduced among 43 cocaine-using individuals on methadone maintenance after an intensive six-month psychosocial treatment including CBT ${ }^{19}$. Negative mood on the Profile of Mood States assessment ${ }^{20}$ showed a linear decrease in 776 individuals with alcohol use disorder during 16-week CBT, with a slight increase near treatment end ${ }^{21}$. However, in a randomized pilot of 77 negative-affect-drinkers participating in 12-week CBT, only those exposed to supplemental affect regulation training experienced reduced PANAS NA scores at posttreatment $(d=.87)^{22}$. Only one study has evaluated PA change during CBT: in 43 cocaineusing adults on methadone, no pre-post PA changes were found after six months of intensive psychosocial treatment containing CBT ${ }^{19}$.

This is the first study to evaluate relationships of baseline and weekly within-treatment NA and PA to cocaine use frequency during outpatient CBT. We pooled data from two randomized controlled trials comparing web-based CBT (CBT4CBT) to cocaine use disorder treatment as usual (TAU). We calculated correlations between baseline NA and PA scores and cocaine use outcomes, and hypothesized that: (1a) baseline cocaine use would be positively correlated with baseline NA and (1b) negatively correlated with baseline PA; and (2a) within-treatment cocaine use would be positively correlated with baseline NA and (2b) negatively correlated with baseline PA. We further hypothesized that (3a) weekly NA would reduce during CBT, consistent with two of three prior studies ${ }^{19,21}$, and (3b) weekly PA would increase during CBT as participants gained new skills. We further hypothesized that lower weekly cocaine use would be associated with (4a) lower NA that week, and (4b) higher PA that week.

\section{Method}

\section{Participants}

Participants were English-speaking adults drawn from two randomized controlled trials of CBT4CBT ${ }^{23,24}$, a computerized cognitive-behavioral treatment for cocaine use disorder. Study 1 participants were recruited from a community outpatient clinic and met criteria for any SUD with some substance use within the past 30 days; these analyses included only those with cocaine dependence $(N=41)$. Study 2 participants $(N=99)$ were recruited from a methadone maintenance clinic, were on a stable methadone dose for a minimum of two months, and met current DSM-IV criteria for cocaine dependence. In both studies, exclusion criteria were minimal (unstable/untreated psychotic disorder, current suicidal or homicidal ideation, inability to read at $6^{\text {th }}$ grade level) to allow for a representative sample of treatment-seeking adults. 


\section{Procedures}

All participants were screened by research assistants and provided written informed consent as approved by the Yale University School of Medicine Human Investigations Committee. A computerized urn randomization program ${ }^{25}$ balanced randomization with respect to gender, ethnicity, primary substance use (Study 1 only), and baseline cocaine use frequency (Study 2 only).

\section{Interventions}

CBT4CBT.-Participants randomized to CBT4CBT were given an individualized log-in code for a password-protected computer in a private clinic room. CBT4CBT was developed to present CBT concepts in an accessible, interactive format and was based on a wellresearched treatment manual ${ }^{18}$. The program presented an introduction, followed by six modules illustrating CBT concepts. As described previously ${ }^{23,24}$, the program included games and interactive exercises to teach skills. Videos for each module presented characters (played by actors) who experienced a common risky situation. After the skill was presented, the characters demonstrated using the skill to avoid substance use. Each module included a review and a homework assignment. A module on HIV risk reduction was available in Study 2.

TAU.-TAU consisted of weekly group and individual drug counseling (Study 1) or weekly group treatment at the methadone clinic and daily methadone dosing (Study 2). See the original reports for further detail ${ }^{23,24}$.

\section{Assessments}

Participants were assessed at baseline, twice each week during treatment, and at study end by independent research assistants. At baseline, the Structured Clinical Interview for DSMIV ${ }^{26}$ was used to assess the presence of SUD and other mental health disorders, and a urine toxicology screen was obtained. During study treatment, participants completed twiceweekly urine toxicology screens. Self-reported cocaine use was assessed at baseline and weekly during treatment using the Substance Use Calendar, similar to the Timeline FollowBack interview ${ }^{27}$. Urine toxicology screens were used as secondary measures; lab samples were tested for cocaine metabolites and data are reported as dichotomous (positive / negative). Affect was assessed at baseline and weekly visits using the 20-item PANAS ${ }^{3}$, which assessed how much the participant felt various positive and negative mood states using a 5-point scale. The PANAS has good test-retest reliability, and convergent and discriminant reliability in individuals with SUD ${ }^{28}$. At all administrations, the PANAS was administered with the timeframe "in the past week" and yielded total NA and PA scales.

\section{Data Analysis}

Studies 1 and 2 were compared on baseline demographic and clinical scores using $F$ tests and chi-square tests to determine whether data could be combined. Correlations between baseline NA and PA, baseline frequency of substance use in past 28 days, and study outcomes were calculated. Bivariate logistic regression tests were used to test whether baseline NA or PA predicted dichotomous indicators of treatment outcome: whether 
individuals completed treatment and were abstinent in the last week, and whether they achieved at least 3 weeks of cocaine abstinence. To examine the relationship between baseline NA and PA scores and cocaine use during treatment, mixed effects models with weekly self-reported cocaine use as the dependent variable and time, treatment condition, their interaction, study, baseline cocaine use frequency, and baseline NA or PA scores were conducted. These and all subsequent mixed models included participant as a random factor with random intercept and a variance components covariance structure.

To examine change in NA and PA during treatment, mixed effects regression models were used with weekly NA or PA scores as the dependent variable and predictor variables including time, treatment condition, their interaction, study, and baseline NA or PA scores. To evaluate whether weekly NA or PA scores were associated with weekly cocaine use, mixed effects models were conducted with weekly cocaine use frequency as the dependent variable and predictor variables including time, treatment condition, their interaction, baseline cocaine use frequency, study, and weekly NA or PA scores as time-varying covariates. For interpretation, a two-tailed alpha level was used in all analyses $(p<.05)$.

\section{Results}

\section{Sample Description}

Baseline characteristics are summarized by study in Table 1 . The combined sample was $57.9 \%$ female $(N=81)$ and the majority were Caucasian $(51.4 \%, N=72)$ with smaller numbers of participants identifying as African-American $(37.1 \%, N=52)$, Hispanic (9.3\%, $N=13)$, Native American $(1.4 \%, N=2)$, or multiracial $(0.7 \%, N=1)$. The majority of participants had a high school education or GED $(70.0 \%, N=98)$ and were unemployed $(75.7 \%, N=106$.) The mean age was $41.81(S D=9.29)$ and participants had been using cocaine for a mean of $12.25(S D=8.74)$ years. Study samples did not differ in duration of cocaine use disorder, lifetime mental health diagnoses, or recent marijuana or cigarette use. Participants in Study 2 were more likely to be Caucasian, on public assistance, or unemployed, and reported more frequent cocaine use and less frequent alcohol use. Rates of treatment completion did not differ. Study 2 participants had fewer self-reported days of abstinence and more cocaine-positive urine toxicology screens.

No study differences were observed in baseline NA scores (Table 1). Mean NA scores for the combined sample $(M=23.07, S D=8.90)$ were similar to other treatment-seeking individuals with SUD ${ }^{29,30}$ although slightly lower than psychiatric inpatients ${ }^{3}$ and individuals with depression ${ }^{31}$.

Participants in Study 2 reported lower baseline PA than participants in Study 1. Participants in Study 2 had mean PA levels similar to other methadone-maintained populations ( $M=25.8$ and 26.4 for two groups, $S D$ not reported ${ }^{32}$ ). Participants in Study 1 had mean PA levels similar to other samples of treatment-seeking individuals with SUD at baseline: $M=31.05$, $S D=8.84^{30}$ and $M=30.00, S D=8.50^{29}$. 


\section{Baseline PANAS NA and PA, Pre-Treatment Cocaine Use and Treatment Outcome}

We first examined the relationship between baseline NA and PA and indicators of pretreatment cocaine use (hypotheses 1a-b). Baseline NA scores were not associated with cocaine-positive urine toxicology screen at baseline $(t(137)=-1.16, p=.25,95 \%$ confidence interval of the difference $[-5.35,1.40])$ nor with cocaine use in the 28 days prior to treatment ( $r=.09, p=.28, n=140)$. Consistent with hypotheses, baseline PA scores were negatively associated with cocaine-positive urine toxicology screen at baseline $(t(136)=$ $3.36, p<.01,95 \%$ confidence interval of the difference $[2.23,8.59])$ and with self-reported cocaine use in the 28 days prior to treatment $(r=-.25, p<.01, n=139)$.

Baseline NA and PA scores were also examined for their relationship to indicators of treatment outcome. Higher baseline NA scores were associated with fewer days retained in treatment $(r=-.25, n=139)$, a shorter maximum duration of cocaine abstinence during treatment $(r=-.20, n=136)$, and more cocaine-positive urine toxicology screens during treatment $(r=.17, n=130$, all $p<.05)$. Similarly, lower baseline NA was associated with completing treatment and being abstinent in the last week ( $\mathrm{B}=-0.07, \mathrm{SE} \mathrm{B}=0.03, \chi^{2}(1, N$ $=123)=5.75, p=.02, \beta=0.93,95 \%$ CI $[0.88,0.99]$ ), although there were no statistically significant baseline NA differences in those who achieved 3 consecutive weeks of cocaine abstinence and those who did not $\left(\mathrm{B}=-0.04, \mathrm{SE} \mathrm{B}=0.02, \chi^{2}(1, N=140)=3.19, p=.07, \beta\right.$ $=0.96,95 \% \mathrm{CI}[0.92,1.00])$. Baseline PA was associated with more days of cocaine abstinence $(r=.33, n=138)$, greater maximum duration of cocaine abstinence $(r=.34, n=$ $135)$, and fewer cocaine-positive urine toxicology screens $(r=-.30, n=129$, all $p<.05)$.

Higher baseline PA scores characterized those who completed treatment and were abstinent in the last week $\left(\mathrm{B}=0.11, \mathrm{SEB}=0.03, \chi^{2}(1, N=122)=14.38, p<.01, \beta=1.12,95 \% \mathrm{CI}\right.$ $[1.06,1.19])$ and those who achieved at least 3 weeks of cocaine abstinence $(\mathrm{B}=0.09$, SE B $=0.03, \chi^{2}(1, N=139)=13.03, p<.01, \beta=1.09,95 \%$ CI $\left.[1.04,1.15]\right)$. Correlations between baseline PANAS and age at first substance use, years of substance use, and days of marijuana or cigarette use in the past 28 days were nonsignificant.

To evaluate whether baseline NA or PA scores were related to self-reported cocaine use during treatment (hypotheses 2a-b) mixed-effects regression analyses were conducted (Table 2). The effects of study were significant, with greater cocaine use in the methadone sample. The effect of time was significant with negative estimates of fixed effects, indicating cocaine use decreased over time. Effects for baseline NA scores were nonsignificant. However, higher baseline PA was associated with less cocaine use during treatment.

\section{Change in NA and PA during Treatment}

To evaluate change in affect during treatment, mixed effects regression models were conducted with weekly NA and PA as the dependent variables and time, treatment condition (CBT vs TAU), their interaction, study, and baseline NA or PA scores as fixed effects and subject as a random effect (Table 2; hypotheses 3a-b). For NA, there was a significant effect for time with a negative fixed effects estimate indicating NA reduced during treatment. Higher baseline NA was correlated with higher NA during treatment. There was no effect for treatment condition. For PA, there was no effect for time or treatment condition, although 
higher PA occurred in the outpatient study and higher baseline PA score was correlated with higher PA during treatment.

\section{Relationship between Weekly NA and PA Score and Weekly Cocaine Use}

Figure 1 presents weekly NA and PA scores for individuals who did or did not establish at least three weeks of cocaine abstinence during treatment. Higher PA scores occurred among those who had at least three weeks of cocaine abstinence. To evaluate the relationship between NA and PA and weekly cocaine use, mixed effects models were conducted with weekly cocaine use frequency as the dependent variable and time, treatment condition, their interaction, study, baseline cocaine use frequency, and weekly NA or PA scores as timevarying covariates and subject as a random factor (Table 3; hypotheses 4a-b). Baseline cocaine use frequency and being in the methadone sample were associated with weekly cocaine use, however, neither time, treatment condition, nor their interaction was associated with weekly cocaine use. Higher weekly PANAS NA scores were associated with weekly cocaine use $(\beta=0.02, \mathrm{SE}=0.01, t(733.85)=2.44, p=.02)$, although weekly PANAS PA scores were not associated with weekly cocaine use.

\section{Discussion}

In this secondary analysis of PANAS scores and their relationship to cocaine use in a combined sample of individuals from two randomized clinical trials of computerized CBT, primary findings were as follows: at baseline, NA scores were high and PA scores were low relative to healthy control samples, but consistent with other substance-using samples. Baseline NA was not associated with cocaine use before or during treatment, although baseline PA was negatively associated with cocaine use before and during treatment. NA decreased during treatment, although PA did not change. When including baseline cocaine use frequency, NA scores, and PA scores, computerized CBT was not associated with reduced cocaine use in contrast to previous trials ${ }^{23}$. As hypothesized, higher weekly NA scores were associated with weekly cocaine use, although no such relationship was found for PA scores.

Baseline PA was negatively associated with cocaine use prior to and during treatment, consistent with other studies $13,15,33$. While causality cannot be inferred, this is consistent with theory that low PA (anhedonia) may be a risk factor for stimulant use disorder ${ }^{10}$. Given the potentially damaging effect of anhedonia, future treatment development might include bolstering CBT by enhancing PA early in treatment, as demonstrated by Stasiewicz ${ }^{22}$ and as suggested by two groups who proposed adding behavioral activation ${ }^{34}$ to reduce anhedonia 8,10 .

During treatment, NA scores reduced, consistent with hypotheses. CBT was not differentially associated with reduced NA, in contrast to findings that NA reduced after CBT with affect regulation training (in simple slope analyses) ${ }^{22}$, an intensive multi-modal treatment including CBT ${ }^{19}$, and a coping CBT module ${ }^{21}$. The computerized CBT in this study did not specifically focus on affect regulation, was less intensive than Magura's protocol that included self-esteem enhancement, and used a smaller sample than Witkiewicz, reducing power to detect interaction effects. 
Although a graph showed that higher weekly PA scores occurred among those who established at least three weeks of cocaine abstinence (Figure 1), the mixed model including baseline cocaine use frequency indicated that during treatment, the level of PA an individual experienced each week did not significantly correlate with their weekly cocaine use (Table 3). It was surprising that distal baseline PA was related to weekly cocaine use in other analyses, but weekly PA fluctuation was not correlated with cocaine use. However, higher weekly NA scores were associated with weekly cocaine use. This may be due to the relationship between cocaine withdrawal and negative mood.

To our knowledge, this is the first study of NA and PA in cocaine use disorder treatment using data from well-controlled randomized controlled trials. Data from two parallel trials increased power and sample heterogeneity, but also may influence generalizability of results: baseline PA levels in each sample were similar to levels in outpatient or methadone samples. Some study effects occurred in mixed models, and the inclusion of individuals from the methadone clinic sample, who were more clinically severe at baseline, may make results less generalizable. Caution should be used in extrapolating findings to non-methadone-using outpatient samples. The level of temporal resolution in these data did not permit testing to see if change in NA or PA temporally preceded cocaine use; however, this is an important question for future studies.

Why might baseline PA matter in cocaine use disorder treatment? Baseline PA was negatively associated with baseline cocaine use, suggesting individuals with higher PA scores may already have been on an upward trajectory. However, baseline PA maintained its negative association with cocaine use during treatment even when including baseline cocaine use. A second possible explanation is that individuals with higher PA were able to avoid substance use via the association of PA scores with a third variable such as reduced craving or desire for drugs ${ }^{29}$, increased abstinence-supportive social support ${ }^{13}$, or self-efficacy for abstinence ${ }^{13}$. This is also consistent with findings that anhedonia was associated with craving in individuals with SUD ${ }^{35}$. Further research that assesses affect concurrently with cocaine use and with craving, social support, or self-efficacy is indicated. If further studies replicate this study's relationship between baseline PA and reduced cocaine use, clinical service providers may need to focus on enhancing PA early in treatment.

\section{Acknowledgements:}

Support for this manuscript came from National Institute on Drug Abuse (Rockville, MD) grants R01-DA 015969 and P50-DA09241 (Clinicaltrials.gov ID number NCT00350610) to Kathleen M. Carroll, PhD. Dr. Decker is supported by New England Mental Illness Research Education and Clinical Center (MIRECC, West Haven, CT) and VA Connecticut Health Care System.

We appreciate the contributions of colleague Elise E. DeVito, PhD, to this manuscript. Portions of this work were presented in poster format at the Association for Behavioral and Cognitive Therapies Annual Meeting in New York, NY on October 30, 2016. The previous poster presentation included correlations between baseline PA and NA scores and cocaine use outcomes, separate mixed models of cocaine use frequency with time-varying NA or timevarying PA that did not include study protocol (not included in this manuscript), and preliminary mixed models on change in NA and PA that did not control for baseline cocaine use severity (not included in this manuscript). This manuscript includes multiple analyses that have not been presented in any other setting or format. 


\section{References}

1. Dutra L, Stathopoulou G, Basden SL, et al. A meta-analytic review of psychosocial interventions for substance use disorders. Am J Psychiatry. 2008;165:179-187. [PubMed: 18198270]

2. Magill M, Ray LA. Cognitive-behavioral treatment with adult alcohol and illicit drug users: a metaanalysis of randomized controlled trials. J Stud Alcohol Drugs. 2009;70:516-527. [PubMed: 19515291]

3. Watson D, Clark L, Tellegen A. Development and validation of brief measures of positive and negative affect: The PANAS Scales. J Pers Soc Psychol. 1988;54:1063-1070. [PubMed: 3397865]

4. Epstein DH, Preston KL. Daily life hour by hour, with and without cocaine: an ecological momentary assessment study. Psychopharmacology. 2010;211:223-232. [PubMed: 20532873]

5. Cheetham A, Allen NB, Yucel M, Lubman DI. The role of affective dysregulation in drug addiction. Clin Psychol Rev 2010;30:621-634. [PubMed: 20546986]

6. Kassel JD, Veilleux JC, Wardle MC, et al. Negative affect and addiction In: al'Absi M, ed. Stress and Addiction: Biological and Psychological Mechanisms. Elsevier; 2007.

7. Volkow ND, Fowler JS, Wang GJ. The addicted human brain viewed in the light of imaging studies: brain circuits and treatment strategies. Neuropharmacology. 2004;47 Suppl 1:3-13. [PubMed: 15464121]

8. Hatzigiakoumis DS, Martinotti G, Giannantonio MD, Janiri L. Anhedonia and substance dependence: clinical correlates and treatment options. Front Psychiatry. 2011;2:10. [PubMed: 21556280]

9. American Psychiatric Association Diagnostic and Statistical Manual of Mental Disorders, 5th edition. Washington, D.C.: Author; 2013.

10. Leventhal AM, Kahler CW, Ray LA, et al. Anhedonia and amotivation in psychiatric outpatients with fully remitted stimulant use disorder. Am J Addict 2008;17:218-223. [PubMed: 18463999]

11. Kilbey MM, Breslau N, Andreski P. Cocaine Use and Dependence in Young-Adults - Associated Psychiatric-Disorders and Personality-Traits. Drug Alcohol Depend. 1992;29:283-290. [PubMed: 1559435]

12. Fredrickson BL, Tugade MM, Waugh CE, Larkin GR. What good are positive emotions in crises? A prospective study of resilience and emotions following the terrorist attacks on the United States on September 11th, 2001. J Pers Soc Psychol. 2003;84:365-376. [PubMed: 12585810]

13. Carrico AW, Woods WJ, Siever MD, et al. Positive affect and processes of recovery among treatment-seeking methamphetamine users. Drug Alcohol Depend. 2013;132:624-629. [PubMed: 23684632]

14. Ryman DH, Biersner RJ, LaRocco JM. Reliabilities and validities of the mood questionnaire. Psychol Rep 1974;35:479-484.

15. Hall SM, Havassy BE, Wasserman DA. Effects of commitment to abstinence, positive moods, stress, and coping on relapse to cocaine use. J Consult Clinical Psychol. 1991;59:526-532. [PubMed: 1918556]

16. Epstein DH, Willner-Reid J, Vahabzadeh M, et al. Real-time electronic diary reports of cue exposure and mood in the hours before cocaine and heroin craving and use. Arch Gen Psychiatry. 2009;66:88-94. [PubMed: 19124692]

17. Petry NM, Alessi SM, Ledgerwood DM. A randomized trial of contingency management delivered by community therapists. J Consult Clin Psychol. 2012;80:286-298. [PubMed: 22250852]

18. <j/>Carroll K. A cognitive-behavioral approach: Treating cocaine addiction. Rockville, MD: U.S. Department of Health and Human Services, National Institutes of Health; 1998.

19. Magura S, Rosenblum A, Lovejoy M, et al. Neurobehavioral treatment for cocaine-using methadone patients: a preliminary report. J Addict Dis 1995;13:143-160.

20. McNair DM, Lorr M, Droppleman LF. POMS: Profile of Mood States. North Tonawanda, NY: Multi-Health Systems; 1992.

21. Witkiewicz K, Bowen S, Donovan DM. Moderating effects of a craving intervention on the relation between negative mood and heavy drinking following treatment for alcohol dependence. J Consult Clin Psychol. 2011;79:54-63. [PubMed: 21261434] 
22. Stasiewicz PR, Bradizza CM, Schlauch RC, et al. Affect regulation training (ART) for alcohol use disorders: development of a novel intervention for negative affect drinkers. J Subst Abuse Treat. 2013;45:433-443. [PubMed: 23876455]

23. Carroll KM, Ball SA, Martino S, et al. Computer-assisted delivery of cognitive-behavioral therapy for addiction: a randomized trial of CBT4CBT. Am J Psychiatry. 2008;165:881-888. [PubMed: 18450927]

24. Carroll K, Kiluk B, Nich C, et al. Computer-assisted delivery of cognitive-behavioral therapy: Efficacy and durability of CBT4CBT among cocaine-dependent individuals maintained on methadone. Am J Psychiatry. 2014;171:436-444. [PubMed: 24577287]

25. Stout RL, Wirtz PW, Carbonari JP, DelBoca FK. Ensuring balanced distribution of prognostic factors in treatment outcome research. J Stud Alcohol. 1994;Supplement 12:70-75.

26. $<\mathrm{j} />$ First MB, R.L. S, Gibbon M, Williams JBW. Structured clinical interview for DSM-IV, patient edition. Washington, D.C: American Psychiatric Press; 1995.

27. Robinson SM, Sobell LC, Sobell MB, Leo GI. Reliability of the Timeline Followback for cocaine, cannabis, and cigarette use. Psychology of Addictive Behavior. 2014;28:154-162.

28. Serafini K, Malin-Mayor B, Nich C, Hunkele K, Carroll KM. Psychometric properties of the Positive and Negative Affect Schedule (PANAS) in a heterogeneous sample of substance users. Am J Drug Alcohol Abuse. 2016;42:203-212. [PubMed: 26905228]

29. Schlauch RC, Gwynn-Shapiro D, Stasiewicz PR, Molnar DS, Lang AR. Affect and craving: Positive and negative affect are differentially associated with approach and avoidance inclinations. Addictive Behaviors. 2013;38:1970-1979. [PubMed: 23380493]

30. Heinz AJ, Peters EN, Boden MT, Bonn-Miller MO. A comprehensive examination of delay discounting in a clinical sample of Cannabis-dependent military veterans making a self-guided quit attempt. Exp clin psychopharmacol. 2013;21:55-65. [PubMed: 23379614]

31. Henriques JB, Davidson RJ. Decreased responsiveness to reward in depression. Cognition and Emotion. 2000;14:711-724.

32. Poling J, Rounsaville BJ, Gonsai K, Severino K, Sofuoglu M. The safety and efficacy of varenicline in cocaine using smokers maintained on methadone: A pilot study. Am J Addict 2010;19:401-408. [PubMed: 20716302]

33. McHugh RK, Kaufman JS, Frost KH, Fitzmaurice GM, Weiss RD. Positive affect and stress reactivity in alcohol-dependent outpatients. J Stud Alcohol Drugs. 2013;74:152-157. [PubMed: 23200161]

34. Dimidjian S, Hollon SD, Dobson KS, et al. Randomized trial of behavioral activation, cognitive therapy, and antidepressant medication in the acute treatment of adults with major depression. $\mathrm{J}$ Consult Clin Psychol 2006;74:658-670. [PubMed: 16881773]

35. Janiri L, Martinotti G, Dario T, et al. Anhedonia and substance-related symptoms in detoxified substance-dependent subjects: a correlation study. Neuropsychobiology. 2005;52:37-44. [PubMed: 15942262] 


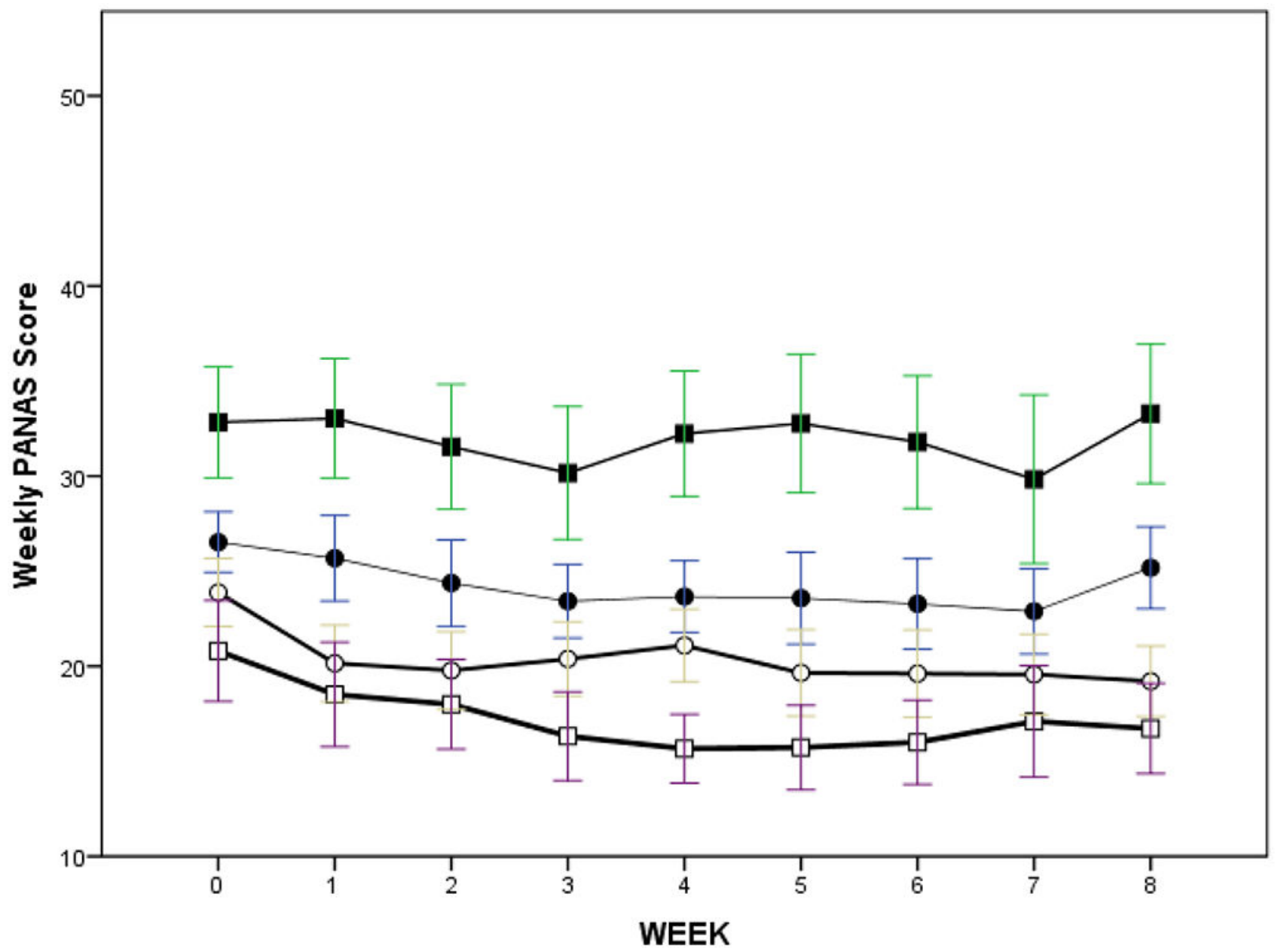

Error Bars: +/- $2 \mathrm{SE}$

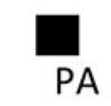

Abstinent
PA Not Abstinent

\section{NA}

Abstinent
O

NA Not Abstinent

Figure 1.

Weekly NA and PA Scores in Individuals who Did or Did Not Establish Three Weeks of Cocaine Abstinence

Note. $N$ for NA $=140,917$ observations. $N$ for PA $=140,912$ observations. 


\section{Table 1}

Sample Description by Study

\begin{tabular}{|c|c|c|c|c|c|c|c|}
\hline & \multicolumn{2}{|c|}{ Study 1} & \multicolumn{2}{|c|}{ Study 2} & \multirow[t]{2}{*}{$t / \chi^{2}$} & \multirow[t]{2}{*}{ df } & \multirow[t]{2}{*}{$p$} \\
\hline & $n$ & $\%$ & $n$ & $\%$ & & & \\
\hline Female & 21 & 51 & 60 & 61 & 1.05 & 1 & .31 \\
\hline \multicolumn{8}{|l|}{ Race and Ethnicity ${ }^{*}$} \\
\hline White & 12 & 29 & 60 & 61 & 12.50 & 4 & .01 \\
\hline African American & 23 & 56 & 29 & 29 & & & \\
\hline Hispanic & 5 & 12 & 8 & 8 & & & \\
\hline Native American & 1 & 2 & 1 & 1 & & & \\
\hline Multiracial & 0 & 0 & 1 & 1 & & & \\
\hline Less than High School Education & 13 & 32 & 29 & 29 & 0.08 & 1 & .78 \\
\hline Married / Committed Relationship & 6 & 15 & 12 & 12 & 0.16 & 1 & .69 \\
\hline Criminal Justice Referral & 13 & 32 & 2 & 2 & 26.71 & 1 & $<.01$ \\
\hline On Public Assistance & 16 & 39 & 77 & 78 & 19.53 & 1 & $<.01$ \\
\hline Unemployed & 16 & 39 & 90 & 91 & 42.45 & 1 & $<.01$ \\
\hline Lifetime Alcohol Use Disorder & 28 & 68 & 71 & 72 & 0.16 & 1 & .69 \\
\hline Lifetime Major Depression & 9 & 22 & 28 & 28 & 0.60 & 1 & .44 \\
\hline Lifetime Anxiety Disorder & 11 & 27 & 32 & 32 & 0.41 & 1 & .52 \\
\hline Antisocial Personality Disorder & 7 & 17 & 7 & 7 & 3.22 & 1 & .07 \\
\hline Study Completer & 26 & 63 & 69 & 70 & 0.53 & 1 & .47 \\
\hline \multirow[t]{2}{*}{ Cocaine-neg. urine at baseline } & 20 & 49 & 17 & 17 & 14.63 & 1 & $<.01$ \\
\hline & $M$ & $S D$ & $M$ & $S D$ & $t$ & df & $p$ \\
\hline Age & 41.22 & 8.76 & 42.05 & 9.53 & -0.48 & 138 & .63 \\
\hline Years of Cocaine Use & 14.13 & 9.06 & 11.46 & 8.53 & 1.65 & 138 & .10 \\
\hline \multicolumn{8}{|l|}{ Days of Drug Use in Past 28 Days } \\
\hline Cocaine & 7.61 & 7.76 & 14.59 & 9.31 & -4.23 & 138 & $<.01$ \\
\hline Alcohol & 4.34 & 7.40 & 0.40 & 0.49 & 5.29 & 138 & $<.01$ \\
\hline Marijuana & 1.66 & 6.10 & 2.53 & 6.42 & -0.74 & 138 & .46 \\
\hline Cigarettes & 23.85 & 8.72 & 25.02 & 8.34 & -0.74 & 138 & .46 \\
\hline Percent Days Abstinent in Study & 82.85 & 18.89 & 60.26 & 30.05 & 4.45 & 134 & $<.01$ \\
\hline Percent Cocaine-positive Urines & 48.45 & 42.77 & 75.39 & 32.69 & -3.91 & 128 & $<.01$ \\
\hline PANAS NA - baseline & 23.93 & 9.19 & 22.72 & 8.80 & 0.73 & 138 & .47 \\
\hline PANAS PA - baseline & 31.35 & 8.98 & 26.94 & 8.31 & 2.77 & 137 & .01 \\
\hline
\end{tabular}

Note.

* $50 \%$ of the cells have expected counts of $<5$, making the statistical value invalid. Bold text denotes statistical significance at $p<.05$. Lifetime alcohol use disorder, major depression, anxiety disorder, and antisocial personality disorder were determined with the Structured Clinical Interview for DSM-IV. PANAS = Positive and Negative Affect Scale. NA = negative affect. PA = positive affect 


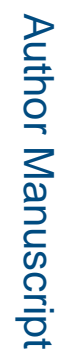

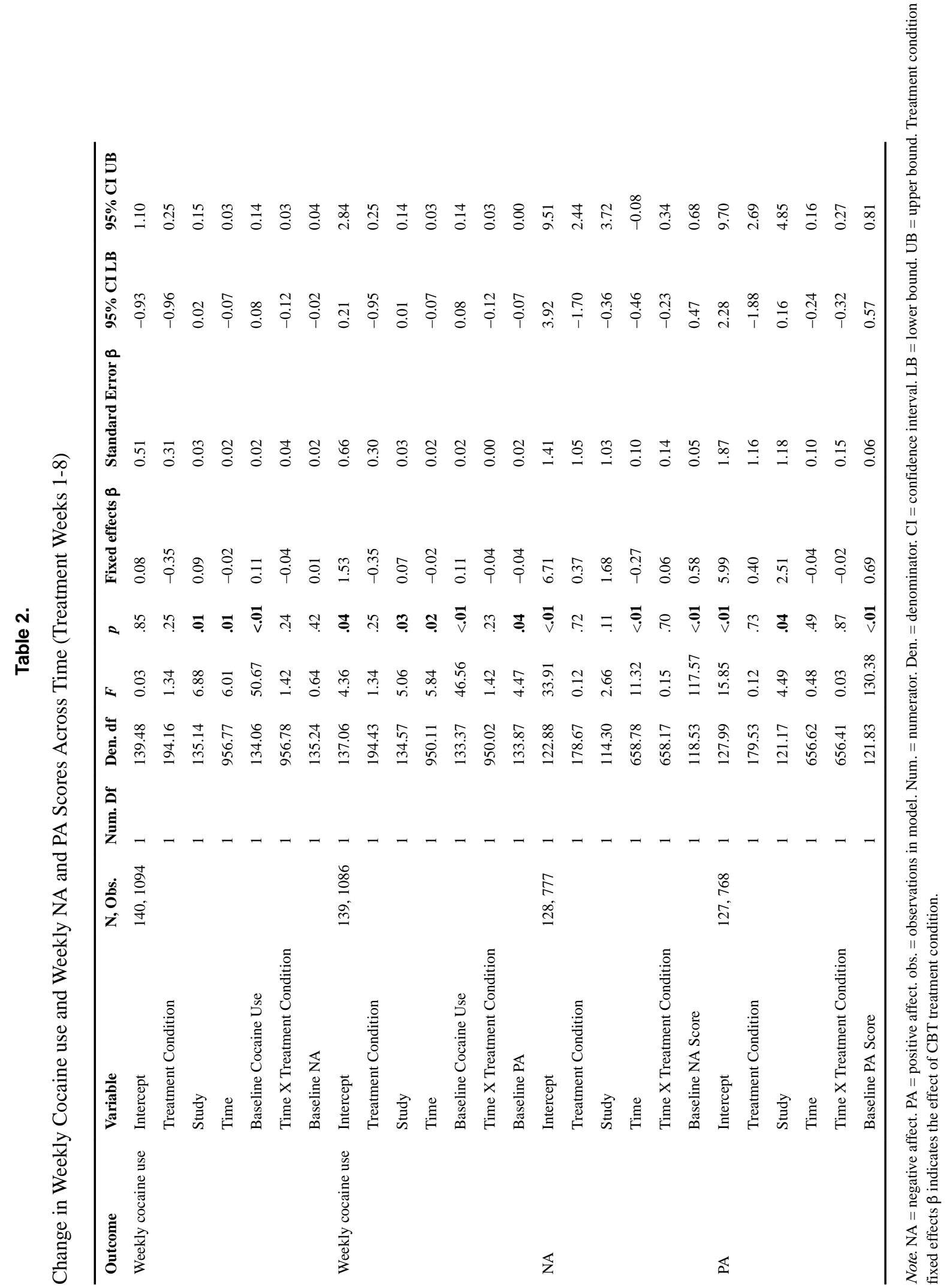




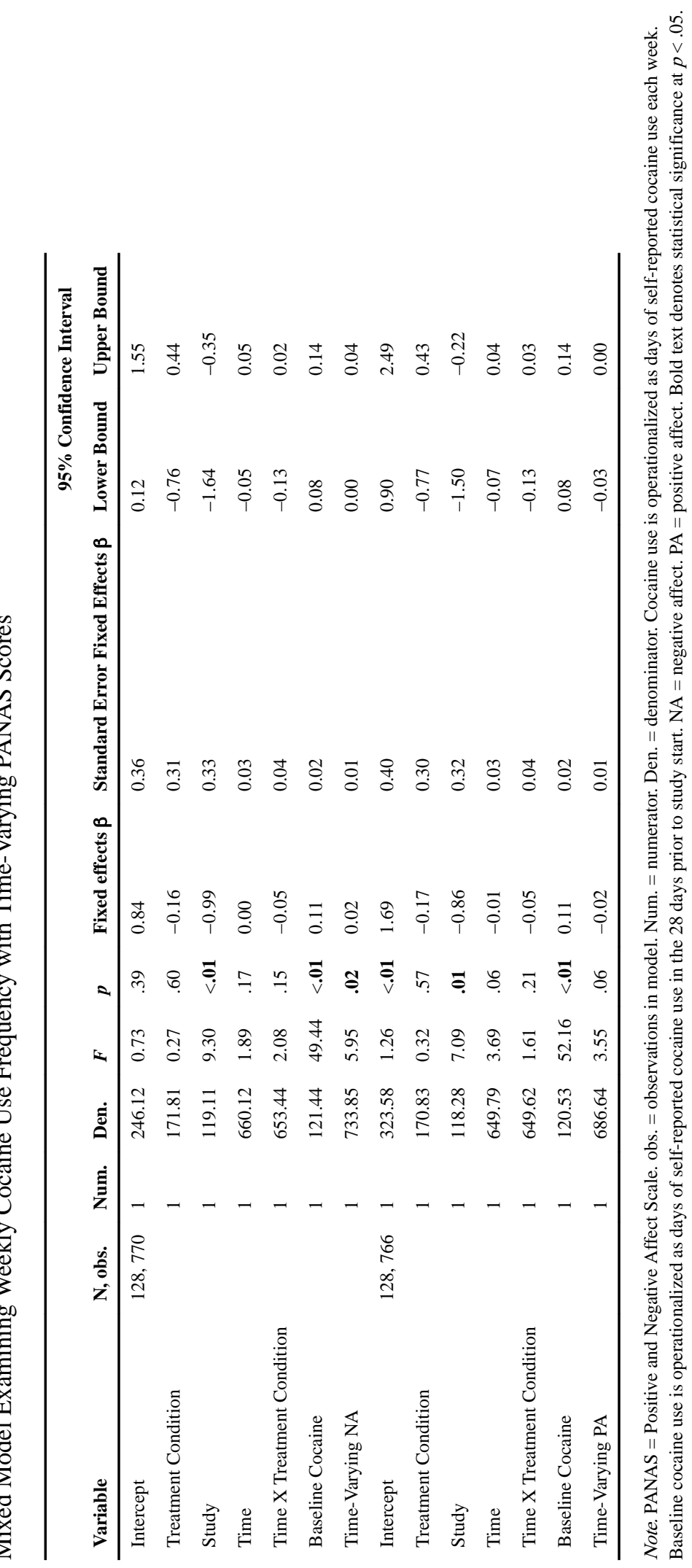

